\title{
Classes of Planar Graphs with Constant Edge Metric Dimension
}

\author{
Changcheng Wei, ${ }^{1}$ Muhammad Salman $\mathbb{D}^{2},{ }^{2}$ Syed Shahzaib, ${ }^{2}$ Masood Ur Rehman $\left(\mathbb{D},{ }^{3}\right.$ \\ and Juanyan Fang ${ }^{1}$ \\ ${ }^{1}$ Institute of Mathematics and Computer Science, Tongling College, Tongling 244000, China \\ ${ }^{2}$ Department of Mathematics, The Islamia University of Bahawalpur, Bahawalpur 63100, Pakistan \\ ${ }^{3}$ Department of Basic Sciences, Balochistan University of Engineering and Technology Khuzdar, Khuzdar 89100, Pakistan
}

Correspondence should be addressed to Masood Ur Rehman; masood@mail.ustc.edu.cn

Received 26 February 2021; Revised 17 March 2021; Accepted 22 March 2021; Published 5 April 2021

Academic Editor: M. Irfan Uddin

Copyright (c) 2021 Changcheng Wei et al. This is an open access article distributed under the Creative Commons Attribution License, which permits unrestricted use, distribution, and reproduction in any medium, provided the original work is properly cited.

\begin{abstract}
The number of edges in a shortest walk (without repetition of vertices) from one vertex to another vertex of a connected graph $G$ is known as the distance between them. For a vertex $x$ and an edge $e=a b$ in $G$, the minimum number from distances of $x$ with $a$ and $b$ is said to be the distance between $x$ and $e$. A vertex $x$ is said to distinguish (resolves) two distinct edges $e_{1}$ and $e_{2}$ if the distance between $x$ and $e_{1}$ is different from the distance between $x$ and $e_{2}$. A set $X$ of vertices in a connected graph $G$ is an edge metric generator for $G$ if every two edges of $G$ are distinguished by some vertex in $X$. The number of vertices in such a smallest set $X$ is known as the edge metric dimension of $G$. In this article, we solve the edge metric dimension problem for certain classes of planar graphs.
\end{abstract}

\section{Introduction}

In graph theory, the metric dimension problem (the problem statement and its background can be found in [1-3]) captivated numerous graph theorists because of its implications in various fields, which includes discovery of networks with security and verification [4], chemistry pharmaceutics in connection with the designing of drug [1], strategies of mastermind games [5], robot navigation [6], problems of coin weighing with its solutions [7], and connected joins in graphs [8]. Due to the empirical significance of this problem, for the last two decades, many researchers have tried to solve this problem by defining several versions of the metric dimension problem such as the fractional version [9], resolving domination [10], the version of doubly metric dimension [11], independent version [12], weighted version [13], the version of $k$-metric dimension [14], solid version [15], mixed version [16], edge version [17], local version [18], simultaneous version [19], connected version [20], and the strong version [8].

This paper is aimed to solve the edge metric dimension problem for five classes of planar graphs $U_{n}$ (class of convex polytopes), $C R_{n}$ (class of chordal ring networks), $H(n)$ (class of $H$-graphs), $P_{1, n}$ (classes of planar graph), and $P_{2, n}$ (classes of planar graph) by investigating their edge metric generators.

\section{The Edge Metric Dimension}

All graphs mentioned in this paper are finite, connected, undirected, and simple. Moreover, the notations $V(G)$ and $E(G)$ will be used for vertex and edge sets of $G$, respectively. The length (number of edges) of a shortest path between two vertices $a$ and $b$ in $G$ is known as the distance, $d(a, b)$, between them. Let $s$ be a vertex and $e=u v$ be an edge in $G$; then, the distance between $s$ and $e$ is $d(e, s)=\min$ $\{d(u, s), d(v, s)\}$. The vertex $s$ distinguishes two distinct edges $e_{1}$ and $e_{2}$ of $G$ if $d\left(s, e_{1}\right) \neq d\left(s, e_{2}\right)$. The metric code of an edge $e$ with respect to a set $X=\left\{x_{1}, x_{2}, \ldots, x_{k}\right\}$ is the $k$-vector:

$$
c_{X}(e)=\left(d\left(e, x_{1}\right), d\left(e, x_{2}\right), \ldots, d\left(e, x_{k}\right)\right) .
$$

The set $X$ is an edge metric generator for $G$ if for every two edges $e_{1}$ and $e_{2}$ of $G$, we have $c_{X}\left(e_{1}\right) \neq c_{X}\left(e_{2}\right)$. The 
cardinality of such a smallest set $X$ for $G$ is called the edge metric dimension of $G$, denoted by $e \operatorname{dim}(G)$ [17].

Let $\mathscr{C}$ be a class of connected graphs $H$ of order $k$. If as $|H|=k \longrightarrow \infty, \quad e \operatorname{dim}(H) \leq M$ for some $M>0$, then we say that the edge metric dimension of $H$ is bounded. Otherwise, it is unbounded. The class $\mathscr{C}$ of graphs is said to have a constant edge metric dimension if the edge metric dimension of graphs in the class is bounded and remains unchanged with the increase in the number of vertices of graphs.

The edge metric dimension problem was introduced by Kelenc et al. in 2018 [17]. They solved this problem for cycle graphs, trees, grid graphs, and complete bipartite graphs. Moreover, examples for the relationships edim $(G)<$ $\operatorname{dim}(G), e \operatorname{dim}(G)=\operatorname{dim}(G)$, and $e \operatorname{dim}(G)>\operatorname{dim}(G)$ were also constructed by them, where the number $\operatorname{dim}(G)$ denoted the metric dimension of $G$ [1]. Further, they explored that the determination of the edge metric dimension for a graph is an NP-complete problem. Later on, this investigation was stretched out by numerous scientists and they added to the writing with an assortment of momentous research work. For readers' interest, we develop a short survey of results about this problem as follows:

(i) Whenever we talk about the ratio $(e \operatorname{dim}(G) / \operatorname{dim}(G))$ between the edge metric dimension and the metric dimension of a graph $G$, we found an example from [17] which illustrates that this ratio is approximately equal to $(5 / 2)$, but this ratio cannot be bounded above in general as proved in [21].

(ii) Graphs of order $n$ having the edge metric dimension $n-1$ are classified in [21], whereas graphs having the edge metric dimension $n-2$ are classified in [22].

(ii) Relationships of edge metric dimension with the maximum and minimum degrees of a graph are developed in $[17,23]$, while the relationship of edge metric dimension with the clique number of a graph is established in [22].

(iv) The edge metric dimension problem of various families and graph operations such as for the barycentric subdivision of Cayley graphs has been solved in [24]. Moreover, the edge metric dimension problem was solved for the join of graphs in $[21,25]$, for the Cartesian product of any graph $G$ with a path $P_{n}$ in [21], and for the lexicographic and corona products of graphs in [25].

(v) Further, the edge metric dimension problem has been solved for various classes of graphs, such as for necklace graphs in [26], for two classes of generalized Peterson graphs $\operatorname{GP}(n, 1)$ and $\mathrm{GP}(n, 2)$ in [23], for web graphs, convex polytope $\mathbb{D}_{n}$, and prism-related graphs in [27], for $n$-sunlet graph and prism graph which turned out to be a constant in [28], for $k$ multiwheel graphs in [29], for two classes of circulant graphs $C_{n}(1,2)$ and $C_{n}(1,3)$ in $[30]$, and for some classes of trees, namely, stars, brooms, double brooms, and banana trees, in [31].

(vi) The edge metric dimension problem via hierarchical product and integer linear programming was discussed in [32].

(vii) Graphs having the smaller edge metric dimension than the metric dimension have been explored in [33]. Further, it has been proved that it is not possible to bound the edge metric dimension of a graph $G$ by some constant factor of the metric dimension of $G$ [33].

(viii) The local version of the edge metric dimension problem has been defined and studied for path graphs, ladder graphs, cycle graphs, star graphs, and wheel graphs in [34].

Kelenc et al. [17] and Filipovic et al. [23] supplied the following results, which are useful tools for the investigation of the edge dimension of graphs.

Proposition 1 (see [17]). Let $G$ be a connected graph and let $\Delta(G)$ be the maximum degree of $G$. Then, e $\operatorname{dim}(G) \geq\left[\log _{2} \Delta\right.$ $(G)]$.

Theorem 1 (see [23]). Let $G$ be a connected graph and let $\delta(G)$ be the minimum degree of $G$. Then, e $\operatorname{dim}(G) \geq 1+$ $\left[\log _{2} \delta(G)\right]$.

Corollary 1 (see [23]). Let $G$ be an $r$-regular graph. Then, $e \operatorname{dim}(G) \geq 1+\left[\log _{2}(r)\right]$.

\section{Classes of Planar Graphs}

In this section, we find the constant edge metric dimension of five classes of planar graphs, namely, family of convex polytopes, family of chordal ring networks, family of $H$ - graphs, and two families of particular planar graphs.

3.1. Class of Convex Polytopes $\mathbf{U}_{\mathbf{n}}$. The graph of convex polytope, $U_{n}$, can be constructed with the combination of the graph of a convex polytope $\mathbb{D}_{n}$ [35] and the graph of a prism $D_{n}$ [36]. The half planar view of this convex polytope is shown in Figure 1. The vertex set of $U_{n}$ is $V\left(U_{n}\right)=\left\{a_{i}, b_{i}\right.$, $\left.c_{i}, d_{i}, e_{i}: 1 \leq i \leq n\right\}$ and the edge set is

$$
\begin{array}{rlr}
E\left(U_{n}\right)=\left\{a_{i} a_{i+1}, b_{i} b_{i+1}, e_{i} e_{i+1}:\right. & 1 \leq i \leq n\} \\
& \bigcup\left\{a_{i} b_{i}, b_{i} c_{i}, c_{i} d_{i}, d_{i} e_{i}, c_{i+1} d_{i}:\right. & 1 \leq i \leq n\},
\end{array}
$$

where the modulo $n$ will be used when the subcript is greater than $n$.

Lemma 1. For $n=2 k+1$ with $k \geq 3$, the set $X=\left\{a_{2}\right.$, $\left.d_{k+1}, e_{1}\right\} \subseteq V\left(U_{n}\right)$ is an edge metric generator.

Proof. Metric codes of the edges of $U_{n}$ with respect to the set $X$ are given as follows: 


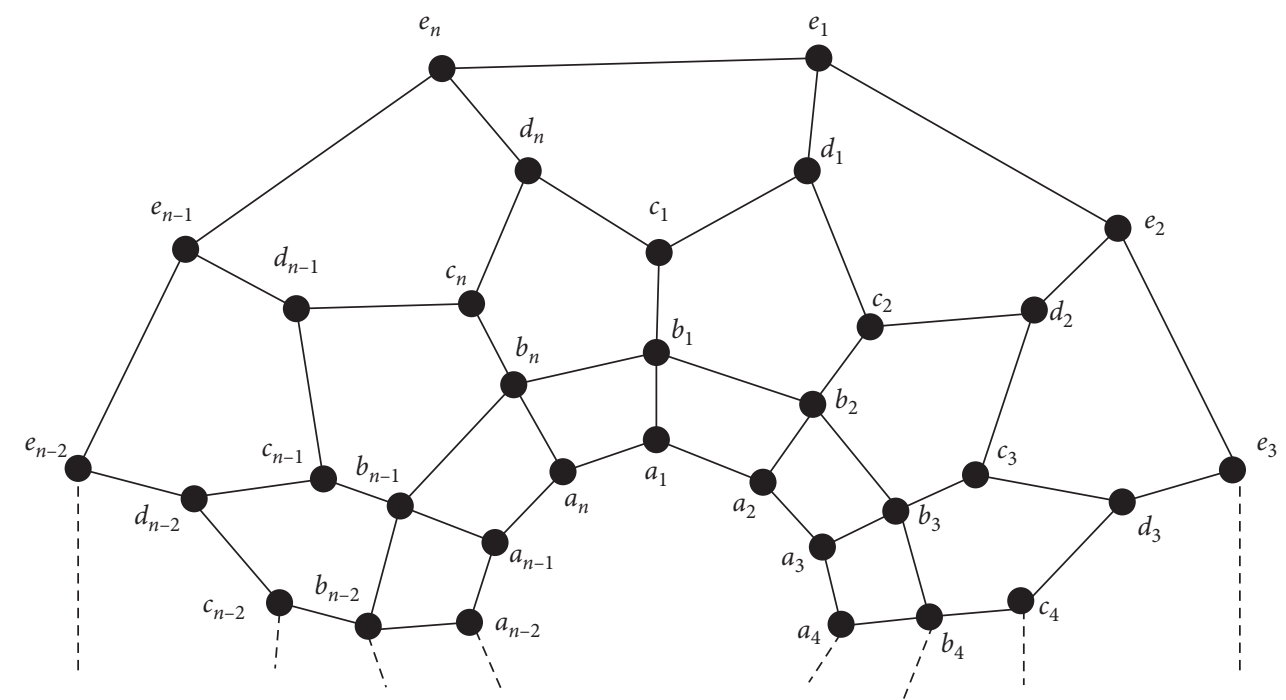

Figure 1: General half view of $U_{n}$ in planar form.

$$
\begin{aligned}
& (0, n-i-7,4), \quad \text { for } i=1, \\
& c_{X}\left(a_{i} a_{i+1}\right)= \begin{cases}(i-2, n-i-7, i+2), & \text { for } 2 \leq i \leq k, \\
(i-2,3, i+2), & \text { for } i=k+1, \\
(n-i+1, i-8, n-i+4), & \text { for } k+2 \leq i \leq n\end{cases} \\
& c_{X}\left(a_{i} b_{i}\right)= \begin{cases}(1, n-i+7,3), & \text { for } i=1, \\
(i-2, n-i+7, i+1), & \text { for } 2 \leq i \leq k+1, \\
(i-2, i-9, i+1), & \text { for } i=k+2, \\
(n-i+2, i-9, n-i+4), & \text { for } k+3 \leq i \leq n,\end{cases} \\
& c_{X}\left(b_{i} b_{i+1}\right)= \begin{cases}(1, n-i-8,3), & \text { for } i=1, \\
(i-1, n-i-8, i+1), & \text { for } 2 \leq i \leq k, \\
(i-1,2, i+1), & \text { for } i=k+1, \\
(i-1, i-9, n-i+3), & \text { for } i=k+2, \\
(n-i+2, i-9, n-i+3), & \text { for } k+3 \leq i \leq n,\end{cases} \\
& c_{X}\left(b_{i} c_{i}\right)= \begin{cases}(2, n-i-7,2), & \text { for } i=1, \\
(i-1, n-i-7, i), & \text { for } 2 \leq i \leq k, \\
(i-1,1, i), & \text { for } k+1 \leq i \leq k+2, \\
(n-i+3, i-9, n-i+3), & \text { for } k+3 \leq i \leq n,\end{cases} \\
& c_{X}\left(c_{i} d_{i}\right)= \begin{cases}(3, n-i-7, i), & \text { for } i=1, \\
(2, n-i-7, i), & \text { for } i=2, \\
(i, n-i-7, i), & \text { for } 3 \leq i \leq k-1, \\
(i, 2, i), & \text { for } i=k, \\
(i, 1, n-i), & \text { for } i=k+1, \\
(n-i+4,3, n-i+2), & \text { for } i=k+2, \\
(n-i+4, i-8, n-i+2), & \text { for } i=k+3,\end{cases}
\end{aligned}
$$

$$
\begin{aligned}
& \{(i, n-i-6, i), \quad \text { for } 1 \leq i \leq k-2 \text {, } \\
& (i, 3, i), \quad \text { for } i=k-1 \text {, } \\
& c_{X}\left(c_{i+1} d_{i}\right)= \begin{cases}(i, 1, i), & \text { for } i=k,\end{cases} \\
& (i, 0, i), \quad \text { for } i=k+1 \text {, } \\
& (i, 2, n-i+2), \quad \text { for } i=k+2 \text {, } \\
& (n-i+3, i-8, n-i+2), \quad \text { for } k+3 \leq i \leq n, \\
& \begin{cases}(3, n-i-8, i-1), & \text { for } i=1, \\
(i+1, n-i-8, i-1), & \text { for } 2 \leq i \leq k,\end{cases} \\
& c_{X}\left(d_{i} e_{i}\right)= \begin{cases}(i+1,0, i-1), & \text { for } i=k+1, \\
(i+1, i-9, n-i+1), & \text { for } i=k+2,\end{cases} \\
& (n-i+4, i-9, n-i+1), \quad \text { for } k+3 \leq i \leq n, \\
& c_{X}\left(e_{i} e_{i+1}\right)= \begin{cases}(4, n-i-9, i-1), & \text { for } i=1, \\
(i+2, n-i-9, i-1), & \text { for } 2 \leq i \leq k+1, \\
(n-i+4, i-10, n-i), & \text { for } k+2 \leq i \leq n .\end{cases}
\end{aligned}
$$

It can be seen that every two distinct edges of $U_{n}$ have different metric codes with respect to $X$, which implies that $X$ is an edge metric generator for $U_{n}$.

Lemma 2. For $n=2 k$ with $k \geq 3$, the set $X=\left\{a_{1}, a_{3}, e_{1}\right\} \subseteq$ $V\left(U_{n}\right)$ is an edge metric generator.

Proof. Metric codes of the edges of $U_{n}$ with respect to the set $X$ are given as follows: 


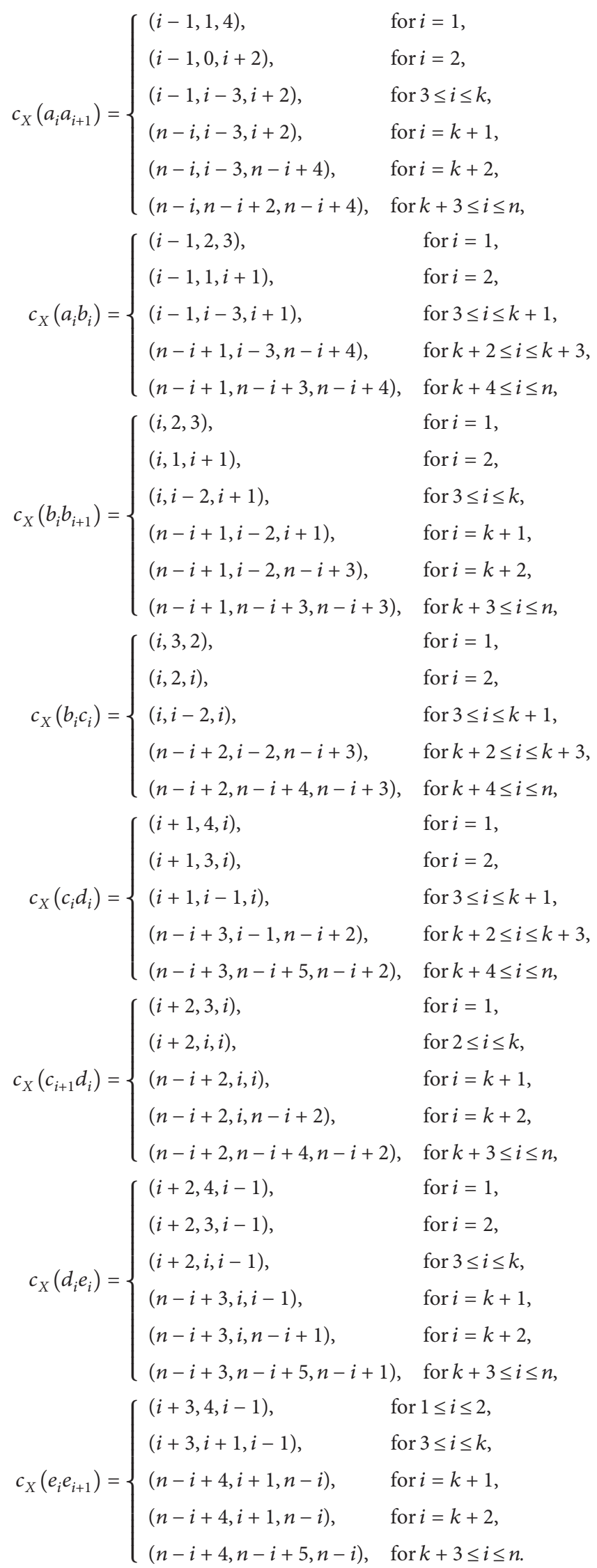

It can be observed that for any two distinct edges $e_{1}$ and $e_{2}, c_{X}\left(e_{1}\right) \neq c_{X}\left(e_{2}\right)$. Therefore, $X$ is an edge metric generator for $U_{n}$.

Theorem 2. For all $n \geq 3$, e $\operatorname{dim}\left(U_{n}\right)=3$.

Proof. Note that the minimum degree in $U_{n}$ is $\delta\left(U_{n}\right)=3$. Therefore, Theorem 2 implies that $e \operatorname{dim}\left(U_{n}\right) \geq 3$. Further, Lemma 4 and Lemma 5 supply that $e \operatorname{dim}\left(U_{n}\right) \leq 3$. Hence, $e \operatorname{dim}\left(U_{n}\right)=3$.

3.2. Class of Chordal Ring Networks. A chord for a path in a graph $G$ is an edge of $G$ which joins the vertices of the path but not a part of that path. A chordal graph is an undirected graph whose every $k$-cycle has a chord for $k \geq 4$. In the literature, numerous types of chordal ring networks can be found, but we consider chordal ring networks supplied by Arden and Lee [37]. These chordal ring networks can be obtained by adding chords in an even order cycle in a regular way. These chords connect an even numbered vertex to an odd numbered vertex [37, 38]. Mathematically, Arden and Lee's purposed chordal ring networks are defined as follows: let a group $Z_{n}$ of integers under addition module $n$, and let three distinct odd elements $a, b$, and $c$ from $Z_{n}$. Then, a chordal ring network of order $n$, denoted by $\mathrm{CR}_{n}(a, b, c)$, is a graph whose vertices are labeled by the elements of $Z_{n}$, and the $i$ th labeled vertex will form an edge with the $(i+a)$ th, $(i+b)$ th, and $(i+c)$ th labeled vertices for each even $i$. Accordingly, it can be seen that these chordal ring networks are cubic bipartite graphs since even numbered vertices are pairwise independent and so are the odd numbered vertices.

In this section, we consider chordal ring networks $\mathrm{CR}_{n}(a, b, c)$ for $a=1, b=3$, and $c=5$, whose one graph is shown in Figure 2. For simplicity, let us divide the vertex set of $\mathrm{CR}_{n}(1,3,5)$ into two $(n / 2)$-element sets as follows:

$$
\begin{aligned}
& U=\left\{u_{(i / 2)}: \text { for } i \in Z_{n} \text { is even }\right\}, \\
& V=\left\{v_{(i-1 / 2)}: \text { for } i \in Z_{n} \text { is odd }\right\} .
\end{aligned}
$$

Accordingly, the edge set of $\mathrm{CR}_{n}(1,3,5)$ should be

$$
E\left(\mathrm{CR}_{n}(1,3,5)\right)=\left\{u_{i} v_{i}, u_{i} v_{i+1}, u_{i} v_{i+2} ; \quad 0 \leq i \leq \frac{n}{2}-1\right\},
$$

where the modulo $n / 2$ will be used on subscripts.

In [39], it has been proved that the family of chordal ring networks $\operatorname{CR}_{n}(1,3,5)$ is planar whenever $n=4 k$ for $k \geq 2$. The planar drawing of this family is shown in Figure 3.

It is an easy task to see that the set $\left\{u_{0}, u_{3}, u_{1}\right\}$ is an edge metric generator for $\mathrm{CR}_{8}(1,3,5)$. For $n>8$ and $n=4 k$, we have the following result.

Lemma 3. For $n=4 k$ with $k \geq 3$, the set $X=\left\{u_{0}\right.$, $\left.v_{(n / 2)-2}, u_{1}\right\} \subseteq V\left(C R_{n}(1,3,5)\right)$ is an edge metric generator.

Proof. Metric codes of the edges of $\mathrm{CR}_{n}(1,3,5)$ with respect to the set $X$ are listed as follows: 


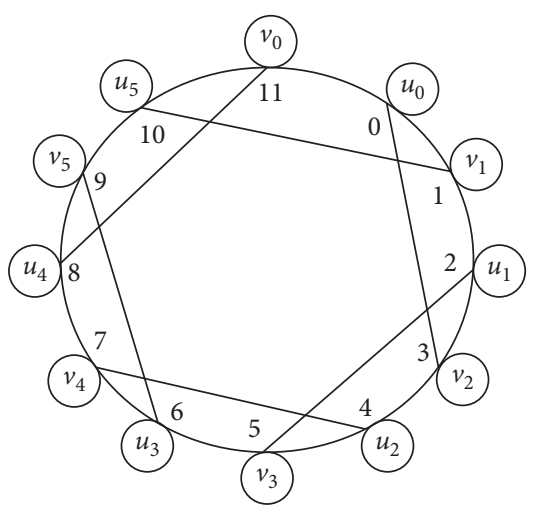

FIGURE 2: The chordal ring network $\mathrm{CR}_{12}(1,3,5)$.

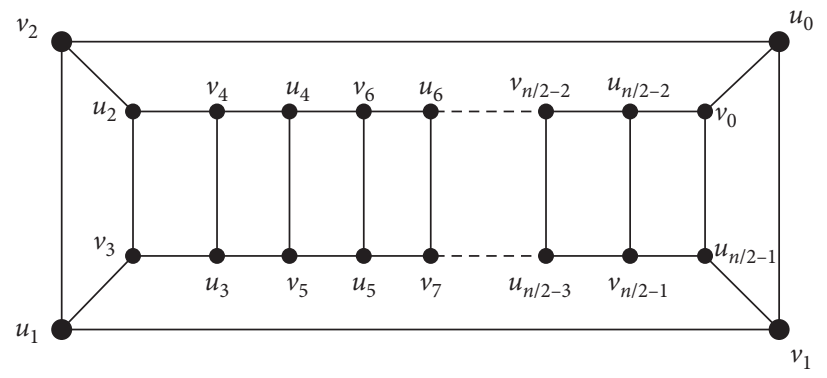

Figure 3: Planar drawing of $\mathrm{CR}_{n}(1,3,5)$ for $n \equiv 0(\bmod 4)$.

$$
\begin{aligned}
& \text { For } i=0, \quad c_{X}\left(u_{i} v_{i}\right)=(0, i+2,0) \text {, } \\
& \text { for } i=1, \quad c_{X}\left(u_{i} v_{i}\right)=(i, i+1,0) \text {, } \\
& \text { for } 2 \leq i \leq k-2 \text {, } \\
& c_{X}\left(u_{i} v_{i}\right)= \begin{cases}(i-1, i+2, i-1), & \text { when } i \equiv 0(\bmod 2), \\
(i, i+1, i-2), & \text { when } i \equiv 1(\bmod 2),\end{cases} \\
& \text { for } k-1 \leq i \leq k \text {, } \\
& c_{X}\left(u_{i} v_{i}\right)= \begin{cases}(i-1,2 k-i-1, i-1), & \text { when } i \equiv 0(\bmod 2), \\
(i, 2 k-i-2, i-2), & \text { when } i \equiv 1(\bmod 2),\end{cases} \\
& \text { for } i=k+1 \text {, } \\
& c_{X}\left(u_{i} v_{i}\right)= \begin{cases}(2 k-i, 2 k-i-1, i-1), & \text { when } i \equiv 0(\bmod 2), \\
(2 k-i+1,2 k-i-2, i-2), & \text { when } i \equiv 1(\bmod 2),\end{cases} \\
& \text { for } k+2 \leq i \leq 2 k-2 \text {, } \\
& c_{X}\left(u_{i} v_{i}\right)= \begin{cases}(2 k-i, 2 k-i-1,2 k-i+2), & \text { when } i \equiv 0(\bmod 2), \\
(2 k-i+1,2 k-i-2,2 k-i+1), & \text { when } i \equiv 1(\bmod 2),\end{cases} \\
& \text { for } i=2 k-1, c_{X}\left(u_{i} v_{i}\right)=(2 k-i+1,0,2 k-i+1) \text {, }
\end{aligned}
$$




$$
c_{X}\left(u_{i} v_{i+1}\right)= \begin{cases}(i, i+2,1), & \text { for } i=0, \\ (i, i+2,0), & \text { for } i=1, \\ (i, i+2, i-1), & \text { for } 2 \leq i \leq k-2, \\ (2 k-i, 2 k-i-2, i-1), & \text { for } k-1 \leq i \leq k, \\ (2 k-i, 2 k-i-2,2 k-i+1), & \text { for } i=k+1, \\ (2 k-i, 1,2 k-i+1), & \text { for } i=2 k-1 \leq 2 k-2,\end{cases}
$$

For $i=0, c_{X}\left(u_{i} v_{i+2}\right)=(i, i+3,0)$,

for $1 \leq i \leq k-3$,

$$
c_{X}\left(u_{i} v_{i+2}\right)= \begin{cases}(i, i+3, i), & \text { when } i \equiv 0(\bmod 2), \\ (i+1, i+2, i-1), & \text { when } i \equiv 1(\bmod 2),\end{cases}
$$$$
\text { for } k-2 \leq i \leq k-1 \text {, }
$$

$$
\begin{aligned}
c_{X}\left(u_{i} v_{i+2}\right)= & \begin{cases}(i, 2 k-i-2, i), & \text { when } i \equiv 0(\bmod 2), \\
(i+1,2 k-i-3, i-1), & \text { when } i \equiv 1(\bmod 2),\end{cases} \\
& \text { for } i=k,
\end{aligned}
$$$$
c_{X}\left(u_{i} v_{i+2}\right)= \begin{cases}(2 k-i-1,2 k-i-2, i), & \text { when } i \equiv 0(\bmod 2), \\ (2 k-i, 2 k-i-3, i-1), & \text { when } i \equiv 1(\bmod 2),\end{cases}
$$$$
\text { for } k+1 \leq i \leq 2 k-3 \text {, }
$$$$
c_{X}\left(u_{i} v_{i+2}\right)= \begin{cases}(2 k-i-1,2 k-i-2,2 k-i+1), & \text { when } i \equiv 0(\bmod 2), \\ (2 k-i, 2 k-i-3,2 k-i), & \text { when } i \equiv 1(\bmod 2),\end{cases}
$$$$
\text { for } 2 k-2 \leq i \leq 2 k-1 \text {, }
$$$$
c_{X}\left(u_{i} v_{i+2}\right)= \begin{cases}(2 k-i-1,1,2 k-i+1), & \text { when } i \equiv 0(\bmod 2), \\ (2 k-i, 1,2 k-i), & \text { when } i \equiv 1(\bmod 2) .\end{cases}
$$

It can be noticed that every two distinct edges of $\mathrm{CR}_{n}(1,3,5)$ have distinct metric codes with respect to the set $X$. It follows that $X$ is an edge metric generator for $\mathrm{CR}_{n}(1,3,5)$.

Theorem 3. For $n=4 k$ with $k \geq 2$, e $\operatorname{dim}\left(C R_{n}(1,3,5)\right)=3$.

Proof. Since $\mathrm{CR}_{n}(1,3,5)$ is a 3-regular graph, so edim $\left(\mathrm{CR}_{n}(1,3,5)\right) \geq 3$, by Corollary 1 . Further, Lemma 3 provides that $e \operatorname{dim}\left(\mathrm{CR}_{n}(1,3,5)\right) \leq 3$. Hence, $e \operatorname{dim}\left(\mathrm{CR}_{n}\right.$ $(1,3,5))=3$.

3.3. Class of $\mathbf{H}-$ Graphs. Each graph of this class is denoted by $H(n)$, which consists of $n \geq 2$ copies of $H$-graphs connected in a planar way as shown in Figure 4. Let the vertex set of $H(n)$ be

$$
V(H(n))=\left\{x_{i}, y_{i}, z_{i} ; \quad 1 \leq i \leq 2 n\right\} .
$$

Then, the edge set of $H(n)$ is

$$
\begin{aligned}
E(H(n))= & \left\{x_{i} x_{i+1}, z_{i} z_{i+1} ; \quad 1 \leq i \leq 2 n-1\right\}, \\
& \bigcup\left\{x_{i} y_{i}, y_{i} z_{i} ; \quad 1 \leq i \leq 2 n\right\} \bigcup\left\{y_{2 i-1} y_{2 i} ; \quad 1 \leq i \leq n\right\}, \\
& \bigcup\left\{x_{2 n} x_{1}, z_{2 n} z_{1}\right\} .
\end{aligned}
$$

Lemma 4. For $n \geq 2$, the set $X=\left\{x_{1}, x_{2 n}, z_{2 n}\right\} \subseteq V(H(n))$ is an edge metric generator.

Proof. Metric codes of the edges of $H(n)$ with respect to the set $X$ are given as follows: 


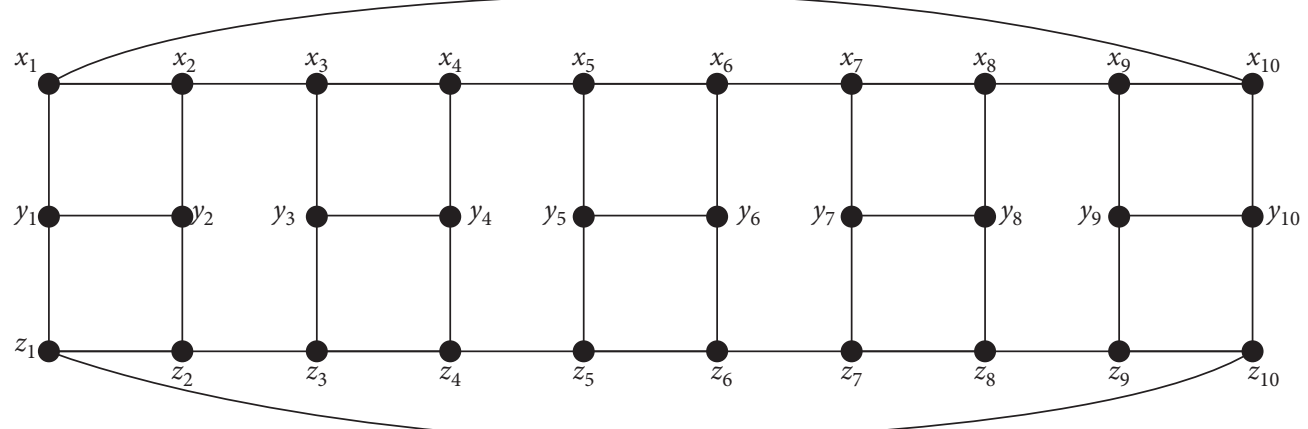

Figure 4: : The $H$-graph $H(10)$.

$$
\begin{aligned}
& c_{X}\left(x_{i} x_{i+1}\right)= \begin{cases}(i-1, i, i+2), & \text { for } 1 \leq i \leq n-1, \\
(i-1, n-i+6, n-i+8), & \text { for } i=n, \\
(n-i+7, n-i+6, n-i+8), & \text { for } n+1 \leq i \leq 2 n-1,\end{cases} \\
& c_{X}\left(x_{2 n} x_{1}\right)=(0,0,2), \\
& c_{X}\left(x_{i} y_{i}\right)= \begin{cases}(i-1, i, i+1), & \text { for } 1 \leq i \leq n, \\
(i-1, n-i+7, n-i+8), & \text { for } i=n+1, \\
(n-i+8, n-i+7, n-i+8), & \text { for } n+2 \leq i \leq 2 n,\end{cases} \\
& c_{X}\left(y_{i} z_{i}\right)= \begin{cases}(i, i+1, i), & \text { for } 1 \leq i \leq n, \\
(i, n-i+8, n-i+7), & \text { for } i=n+1, \\
(n-i+9, n-i+8, n-i+7), & \text { for } n+2 \leq i \leq 2 n,\end{cases} \\
& c_{X}\left(z_{i} z_{i+1}\right)= \begin{cases}(i+1, i+2, i), & \text { for } 1 \leq i \leq n-1, \\
(i+1, n-i+8, n-i+6), & \text { for } i=n, \\
(n-i+9, n-i+8, n-i+6), & \text { for } n+1 \leq i \leq 2 n-1,\end{cases} \\
& c_{X}\left(z_{2 n} z_{1}\right)=(2,0,0),
\end{aligned}
$$$$
c_{X}\left(y_{2 i-1} y_{2 i}\right)= \begin{cases}(2 i-1,2 i, 2 i), & \text { for } 1 \leq i \leq\left\lfloor\frac{n}{2}\right\rfloor, \\ (2 i-1, n-2 i+8, n-2 i+8), & \text { for } i=\left\lceil\frac{n}{2}\right\rceil, \\ (n-2 i+9, n-2 i+8, n-2 i+8), & \text { for }\left\lfloor\frac{n}{2}\right\rfloor+1 \leq i \leq n .\end{cases}
$$

It can be seen that any two different edges of $H(n)$ have different metric codes with respect to the set $X$, which shows that $X$ is an edge metric generator for $H(n)$. 
Theorem 4. For all $n \geq 2$, e $\operatorname{dim}(H(n))=3$.

Proof. It can be seen that $H(n)$ is a 3-regular graph. Therefore, Corollary 1 implies that $e \operatorname{dim}(H(n)) \geq 3$. Further, Lemma 4 yields that $e \operatorname{dim}(H(n)) \leq 3$. Hence, we get the required result.

3.4. Classes of Planar Graph $P_{1, n}$ and $P_{2, n}$. Planar drawings of graphs $P_{1, n}$ and $P_{2, n}$ are shown in Figures 5 and 6 for $n=10$. For $n \geq 3$, the vertex set for both of these planar graphs has the same vertex set, which is

$$
\left\{x_{i}, y_{i}, 1 \leq i \leq n\right\} \bigcup\left\{z_{i}, w_{i}, 1 \leq i \leq n-1\right\} .
$$

But their edge sets are

$$
\begin{aligned}
E\left(P_{1, n}\right)= & \left\{x_{i} x_{i+1}, w_{j} w_{j+1}, \quad 1 \leq i \leq n-1,1 \leq j \leq n-2\right\}, \\
& \bigcup\left\{x_{i} y_{i}, z_{j} w_{j}, \quad 1 \leq i \leq n, 1 \leq j \leq n-1\right\}, \\
& \bigcup\left\{z_{i} y_{i}, z_{i} y_{i+1}, \quad 1 \leq i \leq n-1\right\}, \\
E\left(P_{2, n}\right)= & E\left(P_{1, n}\right) \bigcup\left\{y_{i} y_{i+1}, \quad 1 \leq i \leq n-1\right\} .
\end{aligned}
$$

It is straightforward to see that the set $\left\{x_{1}, y_{3}\right\}$ is an edge metric generator for $P_{1,3}$, which implies that $e \operatorname{dim}\left(P_{1,3}\right) \leq 2$. Further, Theorem 2 provides that $e \operatorname{dim}\left(P_{1,3}\right) \geq 2$ because $\delta\left(P_{1,3}\right)=2$.

Lemma 5. For $n \geq 4$, the set $X=\left\{x_{1}, y_{n}, w_{1}\right\} \subseteq V\left(P_{1, n}\right)$ is an edge metric generator.

Proof. Metric codes of the edges of $P_{1, n}$ with respect to the set $X$ are given as follows:

$$
\begin{gathered}
c_{X}\left(x_{i} x_{i+1}\right)= \begin{cases}(i-1, n-i, 3), & \text { for } i=1, \\
(i-1, n-i, i+1), & \text { for } 2 \leq i \leq n,\end{cases} \\
c_{X}\left(x_{i} y_{i}\right)= \begin{cases}(i-1, n-i+1,2), & \text { for } i=1, \\
(i-1, n-i+1, i), & \text { for } 2 \leq i \leq n-1, \\
(i-1,0, i), & \text { for } i=n,\end{cases} \\
c_{X}\left(y_{i} z_{i}\right)= \begin{cases}(i, n-i+2, i), & \text { for } 1 \leq i \leq n-2, \\
(i, 3, i), & \text { for } i=n-1, \\
(i, 1, i), & \text { for } i=n,\end{cases} \\
c_{X}\left(z_{i} y_{i+1}\right)= \begin{cases}(i+1, n-i+1, i), & \text { for } 1 \leq i \leq n-2, \\
(i+1,2, i), & \text { for } i=n-1, \\
(i+1,0, i), & \text { for } i=n,\end{cases} \\
c_{X}\left(z_{j} w_{j}\right)= \begin{cases}(j+1, n-j+1, j-1), & \text { for } 1 \leq j \leq n-1, \\
(j+1,1, j-1), & \text { for } j=n,\end{cases} \\
c_{X}\left(w_{j} w_{j+1}\right)=(j+2, n-j, j-1),
\end{gathered}
$$

It can be seen that every two distinct edges of $P_{1, n}$ have different metric codes with respect to the set $X$, so $X$ is an edge metric generator for $P_{1, n}$.
Lemma 6. For $n \geq 4$, if $S$ is a minimum edge metric generator for $P_{1, n}$, then $|S| \geq 3$.

Proof. As $\delta\left(P_{1, n}\right)=2$, e $\operatorname{dim}\left(P_{1, n}\right)=|S| \geq 2$, by Theorem 2 . Assume that $|S|=2$ and let $S=\{u, v\}$. Without loss of generality, we let $u=x_{1}$. Then, $S=\left\{x_{1}, v\right\}$, and we have four cases to discuss.

Whenever $v=x_{i}$ for $2 \leq i \leq n$. Then,

$$
\begin{aligned}
& \text { For } 2 \leq i \leq n-1, \quad c_{S}\left(y_{i+1} z_{i}\right)=(i+1,2)=c_{S}\left(z_{i} w_{i}\right) \\
& \text { for } i=n c_{S}\left(z_{i-2} y_{i-1}\right)=(i-1,2)=c_{S}\left(y_{i-1} z_{i-1}\right) .
\end{aligned}
$$

Whenever $v=y_{i}$ for $2 \leq i \leq n$. Then,

$$
\begin{aligned}
& \text { For } 2 \leq i \leq n-1, \quad c_{S}\left(y_{i} z_{i-1}\right)=(i, 0)=c_{S}\left(y_{i} z_{i}\right), \\
& \text { for } i=n c_{S}\left(z_{i-2} w_{i-2}\right)=(i-3,3)=c_{S}\left(w_{i-3} w_{i-2}\right) .
\end{aligned}
$$

Whenever $v=z_{i}$ for $2 \leq i \leq n-1$. Then,

For $2 \leq i \leq n-1, \quad c_{S}\left(y_{i+1} z_{i}\right)=(i+1,0)=c_{S}\left(z_{i} w_{i}\right)$.

Whenever $v=w_{i}$ for $2 \leq i \leq n-1$. Then,

$$
\text { For } 2 \leq i \leq n-1, \quad c_{S}\left(z_{i} w_{i}\right)=(i+1,0)=c_{S}\left(w_{i-1} w_{i}\right) .
$$

In all these cases, we get a contradiction of the fact that $S$ is an edge metric generator for $P_{1, n}$. Hence, our supposition is wrong and so $|S| \geq 3$.

Theorem 5. For $n \geq 4$, e $\operatorname{dim}\left(P_{1, n}\right)=3$.

Proof Lemma 5 and Lemma 6 supply the required result.

Lemma 7. For $n \geq 3$, the set $X=\left\{x_{1}, x_{n}, w_{2}\right\} \subseteq V\left(P_{2, n}\right)$ is an edge metric generator.

Proof. Metric codes of the edges of $P_{2, n}$ with respect to the set $X$ are given as follows:

$$
\begin{gathered}
c_{X}\left(x_{i} x_{i+1}\right)= \begin{cases}(i-1, n-i-1,3), & \text { for } 1 \leq i \leq 2, \\
(i-1, n-i-1, i), & \text { for } 3 \leq i \leq n,\end{cases} \\
c_{X}\left(x_{i} y_{i}\right)= \begin{cases}(i-1, n-i, 3), & \text { for } i=1, \\
(i-1, n-i, 2), & \text { for } i=2,\end{cases} \\
c_{X}\left(y_{i} y_{i+1}\right)= \begin{cases}(i, n-i, n), i, i-1), & \text { for } 3 \leq i \leq n, \\
(i, n-i, i-1), & \text { for } 1 \leq i \leq 2,\end{cases} \\
c_{X}\left(y_{i} z_{i}\right)= \begin{cases}(i, n-i+1,2), & \text { for } 3 \leq i \leq n, \\
(i, n-i+1, i-1), & \text { for } i=1,\end{cases} \\
c_{X}\left(z_{i} y_{i+1}\right)= \begin{cases}(i+1, n-i, 2), & \text { for } 2 \leq i \leq n, \\
(i+1, n-i, i-1), & \text { for } i=1,\end{cases} \\
c_{X}\left(z_{j} w_{j}\right)= \begin{cases}(j+1, n-j+1,1), & \text { for } 2 \leq i \leq n, \\
(j+1, n-j+1, j-2), & \text { for } j=1,\end{cases} \\
c_{X}\left(w_{j} w_{j+1}\right)= \begin{cases}(j+2, n-j+1,0), & \text { for } j=1, \\
(j+2, n-j+1, j-2), & \text { for } 2 \leq j \leq n .\end{cases}
\end{gathered}
$$




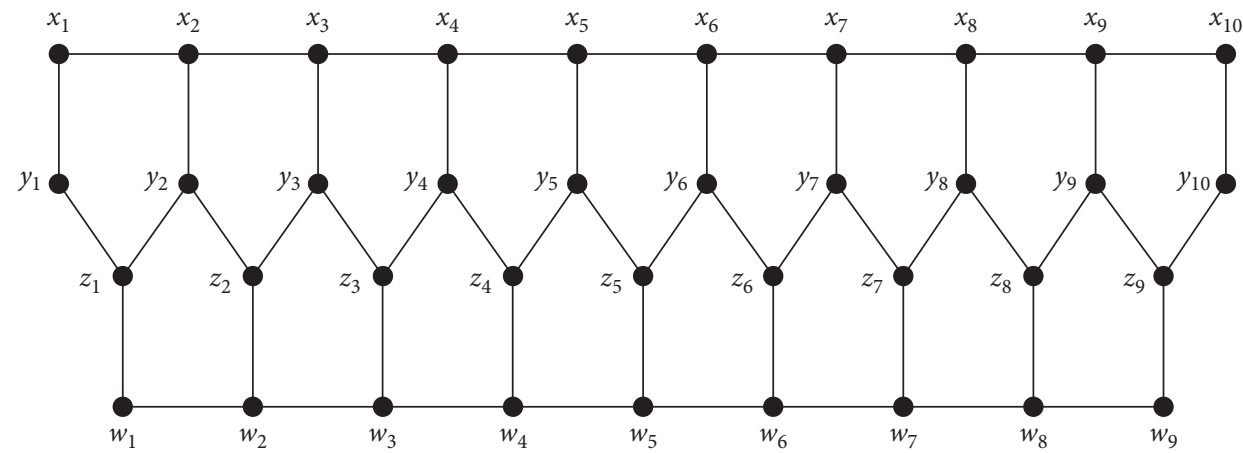

Figure 5: The planar graph $P_{1,10}$.

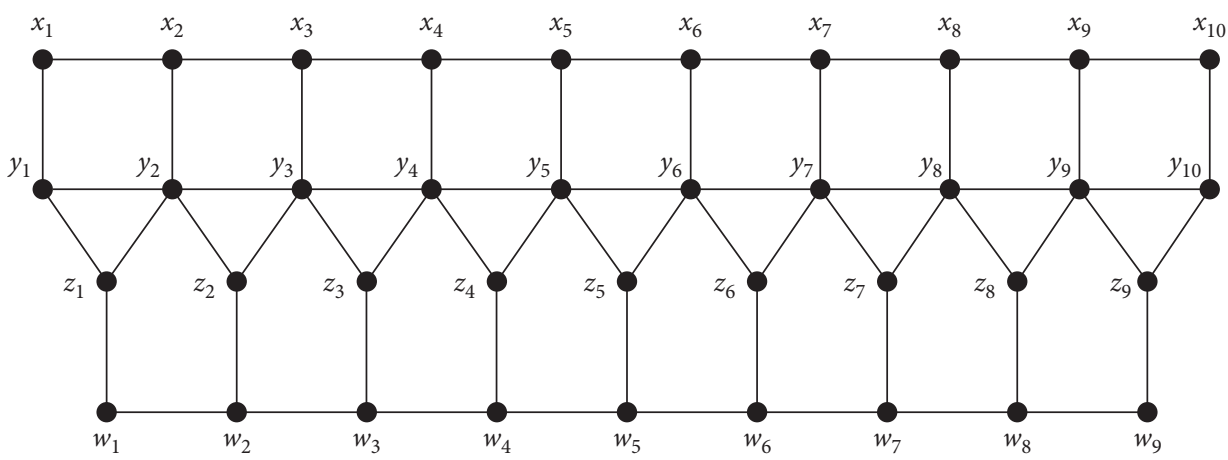

FIgURE 6: The planar graph $P_{2,10}$.

It can be observed that every two distinct edges have different metric codes with respect to the set $X$, so $X$ is an edge metric generator for $P_{2, n}$.

Theorem 8. For $n \geq 3$, e $\operatorname{dim}\left(P_{2, n}\right)=3$.

Proof. Note that the maximum degree in $P_{2, n}$ is $\Delta\left(P_{2, n}\right)=5$. So, Proposition 1 implies that $e \operatorname{dim}\left(P_{2, n}\right) \geq 3$. Further, Lemma 7 yields that $e \operatorname{dim}\left(P_{2, n}\right) \leq 3$.

\section{Conclusion}

With this article, we have extended the study of the edge metric dimension problem towards few classes of planar graphs. We observed that in each of the considered classes of planar graphs, the edge metric dimension is not changed when we increase the number of vertices in graphs, which yields that the investigated edge metric dimension is constant. In fact, we proved that only three vertices are sufficient to distinguish (resolve) every pair of edges in each planar graph of the considered classes [40-44].

\section{Data Availability}

The data used to support the findings of the study are included within the article.

\section{Conflicts of Interest}

The authors declare that there are no conflicts of interest regarding the publication of this paper.

\section{Acknowledgments}

This research was supported by the Anhui Natural Science Research Project (2020) under grant no. KJ2020A0696.

\section{References}

[1] G. Chartrand, L. Eroh, M. Johnson, and O. R. Oellermann, "Resolvability in grpahs and the metric dimnesion of a graph," Discrete Applied Mathematics, vol. 105, no. 1-3, pp. 99-113, 2000.

[2] F. Harary and R. A. Melter, "On the metric dimension of a graph,” Ars Combinatoria, vol. 2, pp. 191-195, 1976.

[3] P. J. Slater, "Leaves of trees," Congressus Numerantium, vol. 14, pp. 549-559, 1975.

[4] Z. Beerliova, F. Eberhard, T. Erlebach et al., "Network discovery and verification," IEEE Journal on Selected Areas in Communications, vol. 24, no. 12, pp. 2168-2181, 2006.

[5] V. Chvátal, "Mastermind," Combinatorica, vol. 3, no. 3-4, pp. 325-329, 1983.

[6] S. Khuller, B. Raghavachari, and A. Rosenfeld, "Landmarks in graphs," Discrete Applied Mathematics, vol. 70, no. 3, pp. 217-229, 1996.

[7] H. Shapiro and S. Soderberg, "A combinatory detection problem," The American Mathematical Monthly, vol. 70, pp. 1066-1070, 1963. 
[8] A. Sebö and E. Tannier, "On metric generators of graphs," Mathematics of Operations Research, vol. 29, pp. 383-393, 2004.

[9] S. Arumugam and V. Mathew, "The fractional metric dimension of graphs," Discrete Mathematics, vol. 312, no. 9, pp. 1584-1590, 2012.

[10] R. C. Brigham, G. Chartrand, R. D. Dutton, and P. Zhang, "Resolving domination in graphs," Mathematica Bohemica, vol. 128, no. 1, pp. 25-36, 2003.

[11] J. Cáceres, C. Hernando, M. Mora et al., "On the metric dimension of cartesian products of graphs," SIAM Journal on Discrete Mathematics, vol. 21, no. 2, pp. 423-441, 2007.

[12] G. Chartrand, V. Saenpholphat, and P. Zhang, "The independent resolving number of a graph," Mathematica Bohemica, vol. 128, no. 4, pp. 379-393, 2003.

[13] L. Epstein, A. Levin, and G. J. Woeginger, "The (weighted) metric dimension of graphs: hard and easy cases," Algorithmica, vol. 72, no. 4, pp. 1130-1171, 2015.

[14] A. Estrada-Moreno, J. A. Rodríguez-Velázquez, and I. G. Yero, "The $k$-metric dimension of a grpah," Applied Mathematics \& Information Sciences, vol. 9, no. 6, pp. 2829-2840, 2015.

[15] A. Hakanen, V. Junnila, and T. Laihonen, "The solid-metric dimension," Theoretical Computer Science, vol. 806, pp. 156-170, 2020.

[16] A. Kelenc, D. Kuziak, A. Taranenko, and I. G. Yero, "Mixed metric dimension of graphs," Applied Mathematics and Computation, vol. 314, pp. 429-438, 2017.

[17] A. Kelenc, N. Tratnik, and I. G. Yero, "Uniquely identifying the edges of a graph: the edge metric dimension," Discrete Applied Mathematics, vol. 251, pp. 204-220, 2018.

[18] F. Okamoto, B. Phinezy, and P. Zhang, "The local metric dimension of a graph," Mathematica Bohemica, vol. 135, no. 3, pp. 239-255, 2010.

[19] Y. Ramírez-Cruz, O. R. Oellermann, and J. A. RodríguezVelázquez, "Simultaneous resolvability in graph families," Electronic Notes in Discrete Mathematics, vol. 46, pp. 241248, 2014.

[20] V. Saenpholphat and P. Zhang, "Connected resolving sets in graphs," Ars Combinatoria, vol. 68, pp. 3-16, 2003.

[21] N. Zubrilina, "On the edge dimension of a graph," Discrete Mathematics, vol. 341, no. 7, pp. 2083-2088, 2018.

[22] M. Wei, J. Yue, J. Yue, and X. Zhu, "On the edge metric dimension of graphs," AIMS Mathematics, vol. 5, no. 5, pp. 4459-4465, 2020.

[23] V. Filipovic, A. Kartelj, and J. Kratica, "Edge metric dimension of some generalized petersen graphs," Results in Mathematics, vol. 74, pp. 1422-6383, 2019.

[24] Z. Mufti, M. Nadeem, A. Ahmad, and Z. Ahmad, "Computation of edge metric dimension of barcycentric subdivision of cayley graphs," Italian Journal of Pure and Applied Mathematics, pp. 714-722, 2020.

[25] I. Peterin and I. G. Yero, "Edge metric dimension of some graph operations," Bulletin of the Malaysian Mathematical Sciences Society, vol. 43, no. 3, pp. 2465-2477, 2020.

[26] J.-B. Liu, Z. Zahid, R. Nasir, and W. Nazeer, "Edge version of metric dimension and doubly resolving sets of the necklace graph," Mathematics, vol. 6, no. 11, p. 243, 2018.

[27] Y. Zhang and S. Gao, "On the edge metric dimension of convex polytopes and its related graphs," Journal of Combinatorial Optimization, vol. 39, no. 2, pp. 334-350, 2020.

[28] R. Nasir, S. Zafar, and Z. Zahid, "Edge metric dimension of graphs," Ars Combinatoria, vol. 147, pp. 143-156, 2018.
[29] M. S. Bataineh, N. Siddiqui, and Z. Raza, "Edge metric dimension of $k$-multiwheel graph, rocky of mountain," Journal of Math, vol. 50, no. 4, pp. 1175-1180, 2020.

[30] M. Ahsan, Z. Zahid, and S. Zafar, "Edge metric dimension of some classes of circulant graphs," Analele Universitatii "Ovidius" Constanta-Seria Matematica, vol. 28, no. 3, pp. 15-37, 2020.

[31] R. Adawiyah, Dafik, R. Alfarisi, R. M. Prihandini, and I. H. Agustin, "Edge metric dimension on some families of tree," Journal of Physics: Conference Series, vol. 1180, Article ID 012005, 2019.

[32] S. Klavžar and M. Tavakoli, "Edge metric dimension via hierarchical product and integer linear programming," Optimization Letters, 2020.

[33] M. Knor, S. Majstorovic, A. T. M. Toshi, R. Skrekovski, and I. G. Yero, "Graphs with the edge metric dimension smaller than the metric dimension," Applied Mathematics and Computation, vol. 405, Article ID 126076, 2021.

[34] R. Adawiyah, Dafik, R. Alfarisi, R. M. Prihandini, I. H. Agustin, and M. Venkatachalam, "The local edge metric dimension of graph," Journal of Physics: Conference Series, vol. 1543, Article ID 012009, 2020.

[35] M. Baca, "Labellings of two classes of convex polytopes," Utilitas Mathematica, vol. 34, pp. 24-31, 1988.

[36] M. Imran, S. A. Ul Haq Bokhary, and A. Q. Baig, "On families of convex polytopes with constant metric dimension," Computers \& Mathematics with Applications, vol. 60, no. 9, pp. 2629-2638, 2010.

[37] B. W. Arden and H. Hikyu Lee, "Analysis of chordal ring network," IEEE Transactions on Computers, vol. C-30, no. 4, pp. 291-295, 1981.

[38] H. S. M. Coxeter, "Self-dual configurations and regular graphs," Bulletin of the American Mathematical Society, vol. 56, no. 5, pp. 413-456, 1950.

[39] I. Javaid, A. Ismail, and M. Salman, "Labeling of chordal rings," Utilitas Mathematica, vol. 90, pp. 61-75, 2013.

[40] J. A. bondy and U. S. R. Murthy, Graph Theory, SpringerVerlag, London, UK, 2008.

[41] M. Javaid, M. Raza, P. Kumam, and J.-B. Liu, "Sharp bounds of local fractional metric dimensions of connected networks," IEEE Access, vol. 8, pp. 172329-172342, 2020.

[42] A. N. A. Koam and A. Ahmad, "Barycentric subdivision of cayley graphs with constant edge metric dimension," IEEE Access, vol. 8, pp. 80624-80628, 2020.

[43] J.-B. Liu, M. K. Aslam, and M. Javaid, "Local fractional metric dimensions of rotationally symmetric and planar networks," IEEE Access, vol. 8, pp. 82404-82420, 2020.

[44] Z. Raza and N. Siddiqui, "The edge metric dimension of Cayley graphs $\Gamma\left(\mathbb{Z}_{n} \oplus \mathbb{Z}_{2}\right)$ and its barycentric subdivisions," Nonlinear Functional Analysis and Applications, vol. 24, no. 4, pp. 801-811, 2019. 